Kyushu J. Math.

Vol. 60, 2006, pp. 305-316

\title{
ON QUASI-ABELIAN VARIETIES OF KIND $k$
}

\author{
Yukitaka ABE and Takashi UMENO \\ (Received 7 April 2005 and revised 23 May 2005) \\ Dedicated to Professor Hideaki Kazama on his sixtieth birthday
}

\begin{abstract}
Gherardelli and Andreotti defined a quasi-abelian variety of kind $k$. However, this definition is somewhat vague and we do not know the real meaning of the 'kind'. We give an example of a quasi-abelian variety which is of kind $k>0$ but not of kind 0 , in the sense of Gherardelli and Andreotti. We prove that if a quasiabelian variety $X=\mathbb{C}^{n} / \Gamma$ has an ample Riemann form of kind $k$, then it has an ample Riemann form of kind $k^{\prime}$ for any $k^{\prime}$ with $2 k \leqq 2 k^{\prime} \leqq n-m$, where rank $\Gamma=n+m$. Next we consider the pair $(X, L)$ of a quasi-abelian variety $X$ and a positive line bundle $L$ on it. We characterize an extendable line bundle $L$ to a compactification $\bar{X}$ of $X$.
\end{abstract}

\section{Introduction}

Let $X=\mathbb{C}^{n} / \Gamma$ be a toroidal group of $\operatorname{rank} \Gamma=n+m$. It is called a quasi-abelian variety if it satisfies generalized Riemann conditions [8]. Moreover, Gherardelli and Andreotti defined a quasi-abelian variety of kind $k$ in $[8]$. There are other works concerning this; see, for example, [1], [7] and [11]. However, we do not completely understand the meaning of the kind even now.

If we consider the kind in the sense of Gherardelli and Andreotti, a quasi-abelian variety may have two different kinds. In Section 3 we give an example of a quasiabelian variety which is of kind $k>0$, but not of kind 0 . On the other hand, we show that if a quasi-abelian variety $X=\mathbb{C}^{n} / \Gamma$ is of kind $k$, then it is of kind $k^{\prime}$ for any $k^{\prime}$ with $2 k \leqq 2 k^{\prime} \leqq n-m$ (Theorem 4.1).

We see from the above example and theorem that any quasi-abelian variety has the smallest kind. Then we can give a precise definition of the kind for a quasi-abelian variety.

2000 Mathematics Subject Classification: Primary 32M05;

Secondary 32L05.

Keywords and Phrases: quasi-abelian varieties of kind $k$; polarization; extendable line bundles. 
We consider the pair $(X, L)$ of a quasi-abelian variety $X$ and a positive line bundle $L$ on it, which is called a polarized quasi-abelian variety. This polarization gives the fibre bundle structure of $X$ on an abelian variety $A$ determined by a hermitian form of $L$. Replacing the fibres by a product of some number of copies of the onedimensional complex projective space $\mathbb{P}^{1}$, we obtain a compactification $\bar{X}$ of $X$. We study the extendability of $L$ to $\bar{X}$. We prove in Section 6 that $L$ is holomorphically extendable to $\bar{X}$ if and only if $L$ is the pull-back of a theta bundle on $A$. This was discussed in [4] and also in [6]. However, the proof was incomplete. In this paper we complete the proof using a more general approach to topologically trivial holomorphic line bundles, which is valid for any fibration of a toroidal group.

\section{Definitions by Gherardelli and Andreotti}

Let $X=\mathbb{C}^{n} / \Gamma$ be a quotient group of $\mathbb{C}^{n}$ by a discrete subgroup $\Gamma$. It is called a toroidal group if $H^{0}(X, \mathcal{O})=\mathbb{C}$. In this case we have $\operatorname{rank} \Gamma=n+m(1 \leqq m \leqq n)$. Let $\mathbb{R}_{\Gamma}$ be the real linear subspace of $\mathbb{C}^{n}$ spanned by $\Gamma$. We define

$$
\mathbb{C}_{\Gamma}:=\mathbb{R}_{\Gamma} \cap i \mathbb{R}_{\Gamma}
$$

Then $\mathbb{C}_{\Gamma}$ is the maximal complex linear subspace contained in $\mathbb{R}_{\Gamma}$, and we have $\operatorname{dim}_{\mathbb{C}} \mathbb{C}_{\Gamma}=m$. Such a toroidal group $X$ is said to be of type $m$. The following definition of quasi-abelian varieties was first given in [8].

Definition 2.1. A toroidal group $X=\mathbb{C}^{n} / \Gamma$ is said to be a quasi-abelian variety if there exists a hermitian form $\mathcal{H}$ on $\mathbb{C}^{n}$ such that:

(1) $\mathcal{H}$ is positive definite on $\mathbb{C}_{\Gamma}$;

(2) the imaginary part $\mathcal{A}:=\operatorname{Im} \mathcal{H}$ of $\mathcal{H}$ is $\mathbb{Z}$-valued on $\Gamma \times \Gamma$.

We call a hermitian form $\mathcal{H}$ in the above definition an ample Riemann form for $\Gamma$ or for $X$. Let $\mathcal{A}_{\Gamma}:=\left.\mathcal{A}\right|_{\mathbb{R}_{\Gamma} \times \mathbb{R}_{\Gamma}}$. Gherardelli and Andreotti [8] also gave the following definition.

Definition 2.2. A quasi-abelian variety $X=\mathbb{C}^{n} / \Gamma$ of type $m$ is said to be of kind $k$ if there exists an ample Riemann form $\mathcal{H}$ for $\Gamma$ with

$$
\operatorname{rank} \mathcal{A}_{\Gamma}=2(m+k) .
$$

We remark that a quasi-abelian variety $X$ may have two different kinds. We can construct such examples. Furthermore, we have a general statement (see Theorem 4.1). Therefore the kind defined by Gherardelli and Andreotti is not 
determined uniquely. We think that it is not only a property of a quasi-abelian variety $X=\mathbb{C}^{n} / \Gamma$ but also that of an ample Riemann form $\mathcal{H}$ for $\Gamma$. We give the following definition.

Definition 2.3. An ample Riemann form $\mathcal{H}$ for $\Gamma$ is said to be of kind $k$ if $\operatorname{rank} \mathcal{A}_{\Gamma}=$ $2(m+k)$, where $\operatorname{rank} \Gamma=n+m$.

\section{An example not of kind 0}

We have fibration theorems for quasi-abelian varieties; see [1], [7] and [8]. The second author studied these arguments more precisely to characterize quasi-abelian varieties. The following theorem is the characterization of quasi-abelian varieties with ample Riemann forms of kind $k$.

THEOREM 3.1. [11, Theorem 4.2] Let $X$ be an n-dimensional toroidal group of type $m$. Then $X$ is a quasi-abelian variety with an ample Riemann form of kind $k$ if and only if there exists an abelian variety $A$ of dimension $m+k$ with an exact sequence

$$
0 \longrightarrow \mathbb{C}^{k} \times\left(\mathbb{C}^{*}\right)^{n-m-2 k} \longrightarrow X \longrightarrow A \longrightarrow 0 .
$$

We can restate the generalized Riemann conditions (Definition 2.1) more concretely. Let $\mathbb{C}^{n} / \Gamma$ be a toroidal group of type $m$ with a period matrix

$$
P=\left(\gamma_{1}, \ldots, \gamma_{n+m}\right)=\left(I_{n} V\right)
$$

where $I_{n}$ is the unit matrix of degree $n$ and $V$ is an $n \times m$ matrix with $\operatorname{rank}(\operatorname{Im} V)=m$. We consider a hermitian form $\mathcal{H}$ on $\mathbb{C}^{n}$ with $\mathcal{A}:=\operatorname{Im} \mathcal{H} \mathbb{Z}$-valued on $\Gamma \times \Gamma$. Then $\mathcal{H}$ has the representative matrix

$$
H=\left(\mathcal{H}\left(e_{i}, e_{j}\right)\right)_{i, j=1}^{n},
$$

where $I_{n}=\left(e_{1}, \ldots, e_{n}\right)$. We set

$$
E=\left(\mathcal{A}\left(\gamma_{i}, \gamma_{j}\right)\right)_{i, j=1}^{n+m},
$$

which is a skew-symmetric matrix with integral coefficients. We write

$$
E=\left(\begin{array}{cc}
E_{1} & E_{2} \\
-{ }^{\mathrm{t}} E_{2} & E_{3}
\end{array}\right),
$$

where $E_{1}$ is an $n \times n$ matrix and $E_{3}$ is an $m \times m$ matrix. 
THEOREM 3.2. [11, Theorem 3.1] Let $\mathbb{C}^{n} / \Gamma$ be a toroidal group of type $m$ with a period matrix $P=\left(\gamma_{1}, \ldots, \gamma_{n+m}\right)=\left(I_{n} V\right)$.

(1) If $\mathbb{C}^{n} / \Gamma$ is a quasi-abelian variety with an ample Riemann form $\mathcal{H}$, then the skew-symmetric matrix $E$ corresponding to $\mathcal{A}=\operatorname{Im} \mathcal{H}$ satisfies the following conditions:

$$
\begin{gathered}
{ }^{\mathrm{t}} V E_{1} V+{ }^{\mathrm{t}} E_{2} V-{ }^{\mathrm{t}} V E_{2}+E_{3}=0 ; \\
\frac{i}{2}\left({ }^{\mathrm{t}} \bar{V} E_{1} V+{ }^{\mathrm{t}} E_{2} V-{ }^{\mathrm{t}} \bar{V} E_{2}+E_{3}\right)>0 .
\end{gathered}
$$

(2) Conversely, if we have a skew-symmetric $(n+m) \times(n+m)$ matrix $E$ with integral coefficients which satisfies (3.1) and (3.2), then $\mathbb{C}^{n} / \Gamma$ is a quasi-abelian variety with an ample Riemann form $\mathcal{H}$ whose imaginary part has the representative matrix $E$ on $\Gamma \times \Gamma$.

Example. We consider a toroidal group $\mathbb{C}^{4} / \Gamma$ of type 2 with period matrix $P=$ $\left(I_{4} V\right)$, where

$$
V=\left(\begin{array}{cc}
\sqrt{2} i & \sqrt{5} i \\
\sqrt{3} i & \sqrt{7} i \\
\sqrt{5} i & -\sqrt{2} \\
\sqrt{7} & -5 \sqrt{3} i
\end{array}\right)
$$

Put

$$
E=\left(\begin{array}{cc}
E_{1} & E_{2} \\
-{ }^{\mathrm{t}} E_{2} & E_{3}
\end{array}\right)
$$

where

$$
E_{1}=\left(\begin{array}{cccc}
0 & -p & -a & -b \\
p & 0 & -c & -d \\
a & c & 0 & -q \\
b & d & q & 0
\end{array}\right), \quad E_{2}=\left(\begin{array}{cc}
-e & -i^{*} \\
-f & -j \\
-g & -k \\
-h & -\ell
\end{array}\right), \quad E_{3}=\left(\begin{array}{cc}
0 & -r \\
r & 0
\end{array}\right)
$$

and $a, b, c, d, e, f, g, h, i^{*}, j, k, \ell, q, r \in \mathbb{Z}$. Further, put

$$
R_{1}:={ }^{\mathrm{t}} V E_{1} V+{ }^{\mathrm{t}} E_{2} V-{ }^{\mathrm{t}} V E_{2}+E_{3}=\left(\begin{array}{cc}
0 & -r_{1} \\
r_{1} & 0
\end{array}\right)
$$

and

$$
R_{2}:=\frac{i}{2}\left({ }^{\mathrm{t}} \bar{V} E_{1} V+{ }^{\mathrm{t}} E_{2} V-{ }^{\mathrm{t}} \bar{V} E_{2}+E_{3}\right) .
$$


By a straight calculation we have

$$
\begin{aligned}
r_{1}= & 5 a+5 \sqrt{6} b+\sqrt{35} c+15 d-\sqrt{2} g-\sqrt{7} \ell+(\sqrt{15}-\sqrt{14}) p \\
& +(\sqrt{14}+5 \sqrt{15}) q+r+i(-2 a-\sqrt{35} b-\sqrt{6} c-7 d \\
& \left.+\sqrt{5} e+\sqrt{7} f-5 \sqrt{3} h-\sqrt{2} i^{*}-\sqrt{3} j-\sqrt{5} k\right) .
\end{aligned}
$$

Suppose that condition (3.1) in Theorem 3.2 holds. Then $r_{1}=0$, hence

$$
\begin{gathered}
b=c=f=g=i^{*}=\ell=p=q=0, \\
a=7 m, \quad d=-2 m, \quad r=-5 m, \quad j=-5 h, \quad k=e \quad \text { with } m \in \mathbb{Z} .
\end{gathered}
$$

In this case we have

$$
R_{2}=\left(\begin{array}{cc}
\sqrt{2} e+2 \sqrt{21} m & \sqrt{5} e-5 \sqrt{3} h+(14+5 i) m \\
\sqrt{5} e-5 \sqrt{3} h+(14-5 i) m & -5 \sqrt{7} h+7 \sqrt{10} m
\end{array}\right)
$$

and

$$
\operatorname{det}(E)=m^{2}\left(2 e^{2}-35 h^{2}+70 m^{2}\right)^{2} .
$$

Put

$$
\begin{aligned}
D(e, h, m):= & \operatorname{det}\left(R_{2}\right) \\
= & -5 e^{2}+(-5 \sqrt{14}+10 \sqrt{15}) e h-75 h^{2}-14 \sqrt{5} e m+70 \sqrt{3} h m \\
& +(-221+14 \sqrt{210}) m^{2}
\end{aligned}
$$

and

$$
D_{1}(e, m):=\sqrt{2} e+2 \sqrt{21} m .
$$

Since $2 e^{2}-35 h^{2}+70 m^{2} \neq 0$ for any $(e, h, m) \in \mathbb{Z}^{3} \backslash\{0\}$, we see that $\mathbb{C}^{4} / \Gamma$ has an ample Riemann form of kind 1 if and only if

$$
m \neq 0, \quad D>0 \quad \text { and } \quad D_{1}>0 .
$$

We note that

$$
D(-1,0,1)=8.18423 \ldots \text { and } \quad D_{1}(-1,1)=7.75094 \ldots
$$

Hence, $\mathbb{C}^{4} / \Gamma$ is a quasi-abelian variety with an ample Riemann form of kind 1 , as the above conditions are satisfied when $e=-1, h=0$ and $m=1$.

Next we show that $\mathbb{C}^{4} / \Gamma$ does not have an ample Riemann form of kind 0 . If it has such a form, then $\operatorname{det}(E)=0, D>0$ and $D_{1}>0$. In this case $m=0$. However,

$$
D(e, h, 0)=-5 e^{2}+(-5 \sqrt{14}+10 \sqrt{15}) e h-75 h^{2}<0
$$


for any $e, h \in \mathbb{Z}$ with $(e, h) \neq(0,0)$, because

$$
(\sqrt{14}-2 \sqrt{15})^{2}-60<0 .
$$

Therefore $\mathbb{C}^{4} / \Gamma$ has no ample Riemann form of kind 0 .

\section{The smallest kind}

THEOREM 4.1. Let $X=\mathbb{C}^{n} / \Gamma$ be a quasi-abelian variety of type $m$. Suppose that $X$ has an ample Riemann form of kind $k(0 \leqq 2 k \leqq n-m)$. Then for any $k^{\prime}$ with $2 k \leqq 2 k^{\prime} \leqq n-m$ there exists an ample Riemann form of kind $k^{\prime}$ for $X$.

Proof. Let $\mathcal{H}$ be an ample Riemann form of kind $k$. We may assume that $\mathcal{H}$ is positive definite on $\mathbb{C}^{n}$. Suppose $2 k<n-m$, and set $\ell:=n-m-2 k$. We can take a period matrix $P=\left(\gamma_{1}, \ldots, \gamma_{n+m}\right)$ of $\Gamma$ such that

$$
\left.\operatorname{Im}^{t} P H \bar{P}=\left(\begin{array}{ccc}
0 & 0 & D \\
0 & 0 & 0 \\
-D & 0 & 0
\end{array}\right)\right\} \ell \text { rows } \text { with } D=\operatorname{diag}\left(d_{1}, \ldots, d_{m+k}\right),
$$

where $H$ is the representative matrix of $\mathcal{H}, d_{1}, \ldots, d_{m+k}$ are positive integers with $d_{1}\left|d_{2}\right| \cdots \mid d_{m+k}$. Furthermore, there exist a positive integer $d_{m+k+1}$ with $d_{m+k} \mid d_{m+k+1}$ and $\gamma_{n+m+1} \in \mathbb{C}^{n}$ with $\gamma_{n+m+1} \notin \mathbb{R}_{\Gamma}$ such that if we set $P_{1}=$ $\left(P \gamma_{n+m+1}\right)$, then

$$
\left.\operatorname{Im}{ }^{\mathrm{t}} P_{1} H \bar{P}_{1}=\left(\begin{array}{ccc}
0 & 0 & D_{1} \\
0 & 0 & 0 \\
-D_{1} & 0 & 0
\end{array}\right)\right\} \ell-1 \text { rows } \quad \text { with } D_{1}=\operatorname{diag}\left(d_{1}, \ldots, d_{m+k+1}\right)
$$

(see the proofs of Lemma 3.1.8 and Theorem 3.1.16 in [6]). We define

$$
\Gamma_{1}:=\Gamma \oplus \gamma_{n+m+1} \mathbb{Z} \quad \text { and } \quad X_{1}:=\mathbb{C}^{n} / \Gamma_{1} .
$$

There exists a covering map $\sigma_{1}: X \longrightarrow X_{1}$ whose covering transformation group is $\mathbb{Z}$. Then $X_{1}$ is a toroidal group of type $m+1$. By the construction of $X_{1}$ we know that $\mathcal{H}$ is an ample Riemann form for $X_{1}$; consequently $X_{1}$ is a quasi-abelian variety.

If $\ell=1$, then $X_{1}$ is an $n$-dimensional abelian variety and we have nothing to show.

Suppose $\ell \geqq 2$. Since

$$
\operatorname{rank}\left(\left.(\operatorname{Im} \mathcal{H})\right|_{\Gamma_{1} \times \Gamma_{1}}\right)=2(m+k+1)=2(m+1)+2 k,
$$


$\mathcal{H}$ is an ample Riemann form of kind $k$ for $X_{1}$. By Theorem 3.1 there exists an abelian variety $A$ of dimension $m+1+k$ with an exact sequence

$$
0 \longrightarrow \mathbb{C}^{k} \times\left(\mathbb{C}^{*}\right)^{n-m-1-2 k} \longrightarrow X_{1} \stackrel{\tau}{\longrightarrow} A \longrightarrow 0 .
$$

Then $\tau \circ \sigma_{1}: X \longrightarrow A$ is an epimorphism. Let

$$
S:=\sigma_{1}^{-1}(\operatorname{ker} \tau)=\operatorname{ker}\left(\tau \circ \sigma_{1}\right) .
$$

Since $\operatorname{ker} \tau=\mathbb{C}^{k} \times\left(\mathbb{C}^{*}\right)^{n-m-1-2 k}$,

$$
\left.\sigma_{1}\right|_{S}: S \longrightarrow \mathbb{C}^{k} \times\left(\mathbb{C}^{*}\right)^{n-m-1-2 k}
$$

is also a covering map with covering transformation group $\mathbb{Z}$. Then $S=\mathbb{C}^{k+1} \times$ $\left(\mathbb{C}^{*}\right)^{n-m-2(k-1)}$. Thus we obtain an exact sequence

$$
0 \longrightarrow \mathbb{C}^{k+1} \times\left(\mathbb{C}^{*}\right)^{n-m-2(k-1)} \longrightarrow X \stackrel{\tau \circ \sigma_{1}}{\longrightarrow} A \longrightarrow 0 .
$$

By Theorem 3.2, $X$ has an ample Riemann form of kind $k+1$.

By Theorem 4.1 and the example in the previous section, we can give a precise definition of 'kind' for any quasi-abelian variety.

Definition 4.2. Let $X=\mathbb{C}^{n} / \Gamma$ be a quasi-abelian variety of type $m$. Then the kind of $X$ is the smallest integer $k$ with $0 \leqq 2 k \leqq n-m$ such that there exists an ample Riemann form of kind $k$ for $X$.

\section{Polarized quasi-abelian varieties}

Let $X=\mathbb{C}^{n} / \Gamma$ be a quasi-abelian variety of type $m$. We consider a positive line bundle $L$ on $X$. We have $L \cong L_{0} \otimes L_{\rho}$, where $L_{0}$ is a topologically trivial holomorphic line bundle and $L_{\rho}$ is the line bundle given by a theta factor $\rho: \Gamma \times \mathbb{C}^{n} \longrightarrow \mathbb{C}^{*}$. We may assume that $\rho$ is a reduced theta factor of type $(\mathcal{H}, \psi)$. Since $L$ is positive, $\mathcal{H}$ is an ample Riemann form for $\Gamma$ (see [2] and [6, Theorem 3.2.13]). We also assume that $\mathcal{H}$ is of kind $k$. In this case the pair $(X, L)$ is called a polarized quasi-abelian variety of kind $k$.

By a suitable change of variables and generators of $\Gamma$, we can take a period matrix $P$ of $\Gamma$ as

$$
P=\left(\left(\begin{array}{ccc}
w_{11} & \ldots & w_{1, m+k} \\
\ldots & \ldots & \ldots \\
w_{n, 1} & \ldots & \ldots \\
& w_{n, m+k}
\end{array}\right) \quad\left(\begin{array}{ccc}
d_{1} & & \\
& \ddots & \\
& & d_{n-k}
\end{array}\right)\right)
$$


where $d_{i}(i=1, \ldots, n-k)$ are positive integers with $d_{1}|\ldots| d_{n-k}$, and

$$
W^{\prime}:=\left(\begin{array}{ccc}
w_{11} & \ldots & w_{1, m+k} \\
\ldots \ldots & \ldots \ldots & \ldots \ldots \ldots \\
w_{m+k, 1} & \ldots & w_{m+k, m+k}
\end{array}\right)
$$

belongs to the Siegel upper-half space $\mathfrak{S}_{m+k}$ of degree $m+k$. If we set

$$
\begin{aligned}
& W^{\prime \prime}:=\left(\begin{array}{ccc}
w_{m+k+1,1} & \ldots & w_{m+k+1, m+k} \\
\ldots \ldots \ldots & \ldots & \ldots \ldots \ldots \ldots \\
w_{n, 1} & \ldots & w_{n, m+k}
\end{array}\right), \\
& D^{\prime}:=\left(\begin{array}{ccc}
d_{1} & & \\
& \ddots & \\
& & d_{m+k}
\end{array}\right), \quad D^{\prime \prime}:=\left(\begin{array}{lll}
d_{m+k+1} & & \\
& \ddots & \\
& & d_{n-k}
\end{array}\right) \text {, } \\
& P^{\prime}:=\left(\begin{array}{c}
W^{\prime} \\
W^{\prime \prime}
\end{array}\right) \quad \text { and } \quad P^{\prime \prime}:=\left(\begin{array}{cc}
D^{\prime} & 0 \\
0 & \left(\begin{array}{c}
D^{\prime \prime} \\
0
\end{array}\right)
\end{array}\right) \text {, }
\end{aligned}
$$

then we have

$$
P=\left(P^{\prime} P^{\prime \prime}\right)
$$

Any $\gamma \in \Gamma$ can be represented uniquely as

$$
\gamma=P^{\prime} a+P^{\prime \prime} b, \quad a \in \mathbb{Z}^{m+k}, b \in \mathbb{Z}^{n-k} .
$$

Furthermore, we may assume that $\rho$ has the following form with respect to this period matrix $P$ :

$$
\rho(\gamma, x)=\mathbf{e}\left(-{ }^{\mathrm{t}} a z-\frac{1}{2}^{\mathrm{t}} a W^{\prime} a\right)
$$

for $\gamma=P^{\prime} a+P^{\prime \prime} b$ and $x=(z, w) \in \mathbb{C}^{m+k} \times \mathbb{C}^{n-m-k}$, where $\mathbf{e}(\cdot)=\exp (2 \pi i \cdot)$. For these facts we refer to [4], [5] or [6] (originally in [3]).

\section{Compactifications and extendable line bundles}

A toroidal group $X=\mathbb{C}^{n} / \Gamma$ of type $m$ has the standard period matrix $P$ in toroidal coordinates as

$$
P=\left(\begin{array}{ccc}
0 & I_{m} & T \\
I_{n-m} & R_{1} & R_{2}
\end{array}\right),
$$

where $\left(I_{m} \quad T\right)$ is a period matrix of an $m$-dimensional complex torus and $\left(\begin{array}{ll}R_{1} & R_{2}\end{array}\right)$ is a real matrix. Let $\left(z_{1}, \ldots, z_{n}\right)$ be coordinates of $\mathbb{C}^{n}$. The projection $p r$ : 
$\mathbb{C}^{n} \longrightarrow \mathbb{C}^{m},\left(z_{1}, \ldots, z_{n}\right) \longmapsto\left(z_{1}, \ldots, z_{m}\right)$ induces the $\left(\mathbb{C}^{*}\right)^{n-m}$-principal bundle $\sigma: X \longrightarrow \mathbb{T}$, where $\mathbb{T}$ is the $m$-dimensional complex torus with period matrix $\left(I_{m} T\right)$. Compactifying the fibres $\left(\mathbb{C}^{*}\right)^{n-m}$ by $\left(\mathbb{P}^{1}\right)^{n-m}$, we obtain the associated $\left(\mathbb{P}^{1}\right)^{n-m}$ bundle $\bar{\sigma}: \bar{X} \longrightarrow \mathbb{T}$. Stein studied the extendability of any line bundle $L$ on $X$ to $\bar{X}$ in [10].

On the contrary, a fibration of a quasi-abelian variety on an abelian variety depends on the kind of an ample Riemann form (Gherardelli-Andreotti Fibration Theorem). Then we consider a polarized quasi-abelian variety $(X, L)$ of kind $k$. We are interested in the extendability of $L$. This problem was discussed in [4] and in [6], but the argument was incomplete. We complete the proof in this section.

We assume that a polarized quasi-abelian variety $(X, L)$ is as in the previous section. Let $x=\left(x_{1}, \ldots, x_{n}\right)$ be complex coordinates of $\mathbb{C}^{n}$ in expressions (5.1) and (5.2). The projection $\tau_{0}: \mathbb{C}^{n} \longrightarrow \mathbb{C}^{m+k},\left(x_{1}, \ldots, x_{n}\right) \longmapsto\left(x_{1}, \ldots, x_{m+k}\right)$ gives a $\left(\mathbb{C}^{*}\right)^{n-m-2 k} \times \mathbb{C}^{k}$-bundle $\tau: X \longrightarrow A$ on an $(m+k)$-dimensional abelian variety $A$ with period matrix $\left(W^{\prime} D^{\prime}\right)$. Compactifying the fibres, we obtain the associated $\left(\mathbb{P}^{1}\right)^{n-m-k}$-bundle $\bar{\tau}: \bar{X} \longrightarrow A$. We study the extendability of $L$ to $\bar{X}$.

To formulate the problem more precisely, we define a group homomorphism $p: \mathbb{C}^{n} \longrightarrow \mathbb{C}^{m+k} \times\left(\mathbb{C}^{*}\right)^{n-m-2 k} \times \mathbb{C}^{k}$ by

$$
p(x):=\left(x_{1}, \ldots, x_{m+k}, \mathbf{e}\left(x_{m+k+1} / d_{m+k+1}\right), \ldots, \mathbf{e}\left(x_{n-k} / d_{n-k}\right), x_{n-k+1}, \ldots, x_{n}\right) .
$$

Any $\eta \in p(\Gamma)$ acts on $\mathbb{C}^{m+k} \times\left(\mathbb{C}^{*}\right)^{n-m-2 k} \times \mathbb{C}^{k}$ by

$$
\begin{aligned}
\eta \cdot\left(y_{1}, \ldots, y_{n}\right):=\left(y_{1}+\eta_{1}, \ldots, y_{m+k}+\eta_{m+k}, y_{m+k+1} \cdot \eta_{m+k+1}, \ldots,\right. \\
\left.y_{n-k} \cdot \eta_{n-k}, y_{n-k+1}+\eta_{n-k+1}, \ldots, y_{n}+\eta_{n}\right),
\end{aligned}
$$

where $\left(y_{1}, \ldots, y_{n}\right)$ are complex coordinates of $\mathbb{C}^{m+k} \times\left(\mathbb{C}^{*}\right)^{n-m-2 k} \times \mathbb{C}^{k}$. This action of $p(\Gamma)$ is properly discontinuous and fixed point free. It is extendable to an action on $\mathbb{C}^{m+k} \times\left(\mathbb{P}^{1}\right)^{n-m-k}$. Then we have

$$
X \cong\left(\mathbb{C}^{m+k} \times\left(\mathbb{C}^{*}\right)^{n-m-2 k} \times \mathbb{C}^{k}\right) / p(\Gamma)
$$

and $\bar{X} \cong \widehat{X}$, where

$$
\widehat{X}:=\left(\mathbb{C}^{m+k} \times\left(\mathbb{P}^{1}\right)^{n-m-k}\right) / p(\Gamma) .
$$

There exists an embedding $\iota: X \longrightarrow \widehat{X}$ with the following commutative diagram:

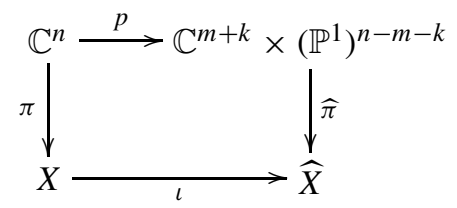


where $\pi$ and $\widehat{\pi}$ are the canonical projections. We see that

$$
\iota(X)=\widehat{\pi}\left(\mathbb{C}^{m+k} \times\left(\mathbb{C}^{*}\right)^{n-m-2 k} \times \mathbb{C}^{k}\right) .
$$

By the projection

$$
\tau_{1}: \mathbb{C}^{m+k} \times\left(\mathbb{P}^{1}\right)^{n-m-k} \longrightarrow \mathbb{C}^{m+k}, \quad\left(y_{1}, \ldots, y_{n}\right) \longmapsto\left(y_{1}, \ldots, y_{m+k}\right),
$$

we obtain a $\left(\mathbb{P}^{1}\right)^{n-m-k}$-bundle $\widehat{\tau}: \widehat{X} \longrightarrow A$. It is isomorphic to $\bar{\tau}: \bar{X} \longrightarrow A$ as fibre bundles on $A$. Then $L \longrightarrow X$ extends holomorphically to $\bar{X}$ if and only if there exists a holomorphic line bundle $L_{1} \longrightarrow \widehat{X}$ such that $\left.\left(\iota^{-1}\right)^{*} L \cong L_{1}\right|_{\iota(X)}$.

Let $\Gamma^{*}:=\tau_{0}(\Gamma)$. Then $A=\mathbb{C}^{m+k} / \Gamma^{*}$. Since $\rho$ has the representation (5.2), we have a theta factor $\rho_{0}: \Gamma^{*} \times \mathbb{C}^{m+k} \longrightarrow \mathbb{C}^{*}$ with $\rho(\gamma, x)=\rho_{0}\left(\tau_{0}(\gamma), \tau_{0}(x)\right.$ ). Therefore $L_{\rho} \cong \tau^{*} L_{\rho_{0}}$, where $L_{\rho_{0}}$ is the theta bundle given by $\rho_{0}$. Hence $L_{\rho}$ is extendable to $\bar{X}$.

Next we treat the topologically trivial holomorphic line bundle $L_{0}$. We suppose that $L_{0}$ extends holomorphically to $\bar{X}$. Then there exists a holomorphic line bundle $\widehat{L} \longrightarrow \widehat{X}$ such that $\left.\left(\iota^{-1}\right)^{*} L_{0} \cong \widehat{L}\right|_{\iota(X)}$.

Lemma 6.1. The line bundle $\widehat{L} \longrightarrow \widehat{X}$ is topologically trivial.

Proof. Let $\widehat{\mathfrak{U}}=\left\{\widehat{U}_{i}\right\}$ be a covering of $\widehat{X}$ consisting of simply connected open sets $\widehat{U}_{i}$. We assume that $\widehat{L}$ is defined by a system of transition functions $\left\{\widehat{g}_{i j}\right\}$ with respect to $\widehat{\mathfrak{U}}$. Define

$$
U_{i}:=\widehat{U}_{i} \cap \iota(X) \quad \text { and } \quad g_{i j}:=\left.\widehat{g}_{i j}\right|_{U_{i} \cap U_{j}} .
$$

Then $\left.\widehat{L}\right|_{\iota(X)}$ is given by a system of transition functions $\left\{g_{i j}\right\}$ with respect to a covering $\mathfrak{U}:=\left\{U_{i}\right\}$ of $\iota(X)$. The Chern class $c_{1}(\widehat{L})$ of $\widehat{L}$ is given by a 2-cocycle $\left\{\widehat{z}_{i j k}\right\} \in Z^{2}(\widehat{\mathfrak{U}}, \mathbb{Z})$ defined by

$$
\widehat{z}_{i j k}:=\frac{1}{2 \pi i}\left(\log \widehat{g}_{i j}+\log \widehat{g}_{j k}-\log \widehat{g}_{i k}\right) .
$$

If we define

$$
z_{i j k}:=\frac{1}{2 \pi i}\left(\log g_{i j}+\log g_{j k}-\log g_{i k}\right),
$$

then $\left\{z_{i j k}\right\}$ determines $c_{1}\left(\left.\widehat{L}\right|_{\iota(X)}\right)$. Since an automorphic factor defining $L_{0}$ is given by an automorphic summand, $L_{0}$ has a system of transition functions which defines the null 2-cocycle (cf. [6, Lemma 2.1.9]). We have $\left.\left(\iota^{-1}\right)^{*} L_{0} \cong \widehat{L}\right|_{\iota(X)}$. Then $\left\{z_{i j k}\right\}=0$. Hence we have $\left\{\widehat{z}_{i j k}\right\}=0$.

LEMMA 6.2. For any non-negative integers $r$, $s$ and $t$ with $r+s+t>0$, we have

$$
H^{q}\left(\mathbb{C}^{r} \times\left(\mathbb{C}^{*}\right)^{s} \times\left(\mathbb{P}^{1}\right)^{t}, \mathcal{O}\right)=0, \quad q>0 .
$$


Proof. By induction on $t$ we can prove the assertion applying the argument used to show $H^{1}\left(\mathbb{P}^{1}, \mathcal{O}\right)=0$ (cf. [9, pp. 47-48]).

Since $\widehat{L}$ is topologically trivial (Lemma 6.1), the pull-back $\widehat{\pi} * \widehat{L}$ is also topologically trivial. By Lemma 6.2 we have

$$
H^{q}\left(\mathbb{C}^{m+k} \times\left(\mathbb{P}^{1}\right)^{n-m-k}, \mathcal{O}\right)=0, \quad q>0 .
$$

Then $\widehat{\pi}^{*} \widehat{L}$ is analytically trivial. Therefore $\widehat{L} \longrightarrow \widehat{X}$ is defined by an automorphic factor

$$
\widehat{\alpha}: p(\Gamma) \times\left(\mathbb{C}^{m+k} \times\left(\mathbb{P}^{1}\right)^{n-m-k}\right) \longrightarrow \mathbb{C}^{*} .
$$

For any $\eta \in p(\Gamma), \widehat{\alpha}(\eta, y)$ does not depend on $y_{m+k+1}, \ldots, y_{n}$. Since

$$
\Gamma^{*} \cong\left(W^{\prime} D^{\prime}\right) \mathbb{Z}^{2(m+k)},
$$

there exists an automorphic factor $\alpha_{0}: \Gamma^{*} \times \mathbb{C}^{m+k} \longrightarrow \mathbb{C}^{*}$ such that

$$
\widehat{\alpha}(\eta, y)=\alpha_{0}\left(\tau_{1}(\eta), \tau_{1}(y)\right) .
$$

$\alpha_{0}$ is equivalent to a homomorphism $\varphi: \Gamma^{*} \longrightarrow \mathbb{C}^{*}$ as $A=\mathbb{C}^{m+k} / \Gamma^{*}$ is an abelian variety. Hence we obtain the following theorem.

THEOREM 6.3. Let $(X, L)$ be a polarized quasi-abelian variety of kind $k$, where $X$ is of type $m$. Let $\tau: X \longrightarrow A$ and $\bar{\tau}: \bar{X} \longrightarrow A$ be as above. Then $L$ extends holomorphically to $\bar{X}$ if and only if there exists a theta bundle $L_{\theta} \longrightarrow A$ such that $L \cong \tau^{*} L_{\theta}$.

Remark. The treatment of topologically trivial holomorphic line bundles in this section is valid for compactification of any fibration of a toroidal group. Using it, we can simplify the argument in [10] and also in [6].

Acknowledgement. The first author was partially supported by Grant-in-Aid for Scientific Research (C)(2)14540163, Japan Society for the Promotion of Science.

\section{REFERENCES}

[1] Y. Abe. Homomorphisms of toroidal groups. Math. Rep. Toyama Univ. 12 (1989), 65-112.

[2] Y. Abe. Lefschetz type theorem. Ann. Mat. Pura Appl. 169 (1995), 1-33.

[3] Y. Abe. Explicit representation of automorphic forms for generalized theta factors. Unpublished, 1997. 
[4] Y. Abe. Meromorphic functions admitting an algebraic addition theorem. Osaka J. Math. 36 (1999), 343-363.

[5] Y. Abe. Construction of automorphic forms for ample factors of quasi-abelian varieties. Kyushu J. Math. 57 (2003), 51-85.

[6] Y. Abe and K. Kopfermann. Toroidal Groups (Lecture Notes in Mathematics, 1759). Springer, Berlin, 2001.

[7] B. Dodson. Fibrations of quasi-abelian varieties. Boll. Un. Mat. Ital., Suppl. 2 (1980), 283-317.

[8] F. Gherardelli and A. Andreotti. Some remarks on quasi-abelian manifolds. Global Analysis and Its Applications. Vol. II. International Atomic Energy Agency, Vienna, 1974, pp. 203-206.

[9] Ph. Griffiths and J. Harris. Principles of Algebraic Geometry. Wiley, New York, 1978.

[10] M. Stein. Abgeschlossene Untergruppen komplexer abelscher Liescher Gruppen. Dissertation, University of Hannover, 1994.

[11] T. Umeno. Period matrices for quasi-abelian varieties. Japan. J. Math. 29 (2003), 117-133.

\author{
Yukitaka Abe \\ Department of Mathematics \\ Toyama University \\ Gofuku, Toyama 930-8555 \\ Japan
}

(E-mail: abe@sci.toyama-u.ac.jp)

Takashi Umeno

Department of Mathematics

Kyushu Sangyo University

Fukuoka 813-8503

Japan

(E-mail: umeno@ip.kyusan-u.ac.jp) 